\title{
versants
}

\section{Gustav Mahler, le dieu rittérien. William Ritter mélomane}

Céline Conus

Université de Lausanne

\begin{abstract}
Gustav Mahler représente sans aucun doute un cas particulier dans la vie et la carrière de critique d'art de William Ritter, contemporain du compositeur autrichien, et qui voit en ce dernier l'avènement d'une nouvelle ère musicale. La conversion de Ritter à la musique de Mahler marque le début d'une extraordinaire passion que le Suisse exposera dans de nombreux écrits de diverse nature (articles, critiques, analyses, correspondance, journal) : autant de sources grâce auxquelles il nous est permis de revivre, presque au jour le jour, la naissance d'une œuvre et l'avènement d'un compositeur qui compte désormais parmi les plus importants de l'histoire de la musique occidentale, acteur incontournable de la transition entre le $\mathrm{XIX}^{\mathrm{e}}$ siècle romantique et le $\mathrm{xx}^{\mathrm{e}}$ siècle moderne.
\end{abstract}

Keywords: Gustav Mahler, William Ritter, symphonie, réception, critique d'art

«Et le soir, ayant assez médité notre impression, nous sifflâmes comme des enragés » (Rp3I, I945 cité par Meylan 2000 : 25)'. C'est ainsi que William Ritter relate la création ${ }^{2}$ de la symphonie $n^{\circ} 4$ de Gustav Mahler à Munich, le 25 novembre 190I. Difficile de croire que ce sont là les mots de celui qui deviendra le plus ardent admirateur et défenseur du compositeur. Quantité d'ouvrages rédigés par les spécialistes de Mahler comptent le nom de Ritter dans leur index : ici une anecdote ou une photo, là une lettre ou un extrait de critique musicale. Rien d'étonnant : le Neuchâtelois est en quelque sorte le premier des mahlériens.

Dans l'autobiographie qu'il dicte à Josef Ritter-Tcherv, son fils adoptif, Ritter donne quelques repères quant à son éducation musicale (Meylan $2000: 432-442)$. On apprend ainsi qu'il débute un « apprentissage sérieux de la musique » en I88I, qu'il écrit ses « premières chroniques de concert » en I883 et qu'il fait en I 886 un " pèlerinage à Bayreuth ». Sans entrer dans les détails, précisons que Wagner est en effet une des première passions musicales de Ritter (voir Vogel 20I9) : «Il a été en art le Dieu de ma première communion $»^{3}$. Ainsi, on peut se demander ce que contient le dossier que Ritter

I Nous utilisons le code de classement adopté par Claude Meylan (2000). Pour les textes : $\mathrm{Rp}$ (écrits publiés), Ri (écrits inédits). Pour la correspondance : RM (Ritter à Mahler) et MR (Mahler à Ritter).

2 Dans le jargon musical, la «création » d'une œuvre désigne sa première exécution publique.

3 Willam Ritter, Chroniques musicales étrangère. Écho de Bayreuth, date inconnue, pI, cité par Laura Vogel (2018). 
a intitulé « Ma musique avant Bayreuth $»^{4}$ qui semble tracer une ligne de démarcation entre l'avant et l'après Wagner. Quoi qu'il en soit, notons que l'esthétique wagnérienne a profondément marqué Ritter. Dans les Études d'art étranger, il donne quelques précisions supplémentaires : «Pendant mes trois odieuses années d'internat, on m'avait donné des leçons de piano comme on sait les donner en province de France (Ritter I906 : 4I5) ». Plus loin, il ajoute :

\begin{abstract}
Rentré à la lumière de la vie familiale et de ses douces lampes à huile [...] on me mit à Clementi. La cure fut radicale. Un à un j'entrou'vris moi-même Haydn, Mozart, Beethoven, Mendelssohn, Schumann, Brahms ; les concerts d'abonnement m'initièrent à la symphonie et aux grands solistes [...]. Je potassais les livres de théorie et les biographies d'artistes comme un beau diable. Deux ans après, dans un des journaux du pays, celui qui a le plus d'abonnés, j'étais critique musical attitré, celui que le petit chef-lieu traite de gamin, mais discute avec fureur ou sympathie protectrice, celui qui casse toutes les vitres, qui ne recule devant aucune audace, et dont le nom suffit à faire dindonner les gens sérieux (Ritter 1906 : 416).
\end{abstract}

En I888, il entreprend un « projet de livre sur la musique scandinave » et se rend à Vienne où il effectue des études musicales plus poussées : «Inscription à l'Université (cours d'harmonie d'Anton Bruckner, d'histoire de la musique d'Eduard Hanslick) "5. Il s'agit là de deux des plus influents personnages du paysage musical d'alors. Hanslick est un critique très écouté et Bruckner, éminent compositeur, grand admirateur de Wagner et professeur au Conservatoire de Vienne, compte un certain Gustav Mahler parmi ses élèves. On peut néanmoins douter que Ritter ait été l'élève de Bruckner au même titre que Mahler. Il est plus probable qu'il ait suivi des cours collectifs publics donnés par le maître ${ }^{6}$. Retenons donc que si Ritter n'a pas encore écouté la musique de Mahler, il en a forcément entendu parler.

Dès I90I, année de la première audition par le Neuchâtelois d'une œuvre de Mahler, les créations du compositeur viennois rythment la vie de Ritter. Fasciné, ce dernier organise ses voyages selon les concerts, insiste pour être présent lors des répétitions et s'efforce de ne manquer aucune première audition : "Quand Mahler vivait, à chaque première d'une nouvelle symphonie nous courions le rejoindre et passer auprès de lui les quelques jours des grandes répétitions d'ensemble » (Rp28, I9I3 cité par Meylan 2000 : 95). Il lui

4 Archives littéraires suisses, boîte $n^{\circ}$ 26, cote A-3-b.

5 Remarquons qu'il n'est question que de théorie musicale et non de pratique instrumentale. On sait que la mère de Ritter jouait du piano et que lui-même se mit à cet instrument à l'âge de quinze ans.

6 C'est en tous les cas ce que semblent confirmer ces mots, tirés d'un article intitulé « Souvenirs sur Anton Bruckner ", publié posthumément dans la revue suisse Feuilles musicales, IO, 1956, pp. I89-19I : « Les Viennois ne sont pas fréquents à un cours d'harmonie populaire, ils sont tous au Conservatoire, eux » (190). 
faut désormais assister à la naissance de chacune des œuvres de Mahler : "Son emprise sur moi déjà prenait le caractère d'une possession » (Rp3I, I945 cité par Meylan 2000 : 30). Il est comme aspiré par cet espace sans limite qui s'ouvre soudain devant le monde, cette sorte de trou noir musical si dense qu'il attire tout à lui, irrémédiablement. L'année 1902 marque le début de la correspondance avec le Maître. La rencontre " fatale et inévitable " (Rp3I, I945 cité par Meylan 2000 :3I) a lieu en 1906.

Situons brièvement Mahler dans le contexte musical de ce xix ${ }^{e}$ siècle finissant ${ }^{7}$. Certains musicologues y voient « l'esthétique [...] d'un romantisme qui n'en finit pas d'agoniser » dans laquelle « on retrouve souvent la nostalgie [...] d'une culture qui sent sa fin approcher » (Beltrando-Patier 2004: 910). La musique romantique tente donc de se dépasser, hantée par les figures tutélaires de Brahms et de Wagner. Mahler met pourrait-on dire un terme à cette agonie, mais en rendant cette mort grandiose et féconde. Ritter le dit avec, comme à son habitude, une certaine pompe : «C'est l'oraison funèbre du passé prononcée par le surhomme de demain » (Ritter 1906: 283).

Mais la musique de Mahler, dans toute son inventivité, n'est-elle pas également la preuve de la vitalité extraordinaire de la production de cette époque ? Les idées fusent, de nombreuses modulations s'enchaînent, transportant l'auditeur dans des "symphonies-monde ", comme les appelait Mahler, un paysage sonore tantôt connu tantôt incongru, un organisme qui n'en finit pas de muter et de gonfler, jusqu'à saturation, jusqu'au paroxysme de l'expression romantique. Ce que permet Mahler, c'est la transition entre deux mondes. En synthétisant la première école de Vienne (Haydn, Mozart, Schubert, Beethoven), les grandes figures romantiques (Bruckner, Brahms, Mendelssohn ou Liszt) et l'esthétique wagnérienne, qui n'épargna alors rien ni personne, il dévoile un passage vers le $\mathrm{xx}^{\mathrm{e}}$ siècle. Il joue ainsi un rôle essentiel dans la naissance de la seconde école de Vienne (Schönberg, Berg, Webern) qui explore l'atonalité et ouvre la voie à la musique dite contemporaine. Ainsi, pour Pierre Boulez ${ }^{8}$, Mahler est ce " compositeur Janus », une face tournée vers le passé, l'autre vers le futur, celui qui « dynamite l'univers tonal »`. Il aura fallu presque un siècle pour qu'advienne cette véritable révolution. Là encore, Ritter semble l'avoir senti :

7 Gustav Mahler (I860-I9II) écrit ses symphonies entre I888 et I9Io. La I ${ }^{\text {ère }}$ symphonie est créée à Budapest le 20 novembre 1889 . La symphonie $n^{\circ} 9$ est créée de façon posthume le 14 octobre 1924, à Vienne. On en compte une $\mathrm{IO}^{\mathrm{e}}$, non achevée. Pour un catalogue complet, se référer notamment à l'ouvrage de l'éminent mahlérien Henry-Louis de La Grange (2007).

8 Pierre Boulez (1925-20I6), compositeur et chef d'orchestre français.

9 Mahler, autopsie d'un génie, documentaire, Andy Sommer, diffusé sur Arte le I9 mai 2oII, [partiellement en ligne] : https://www.youtube.com/watch?v=NVoMtan_wz4, consulté le 30 septembre 2018. 
Au lieu de voir dans les anciens le style, la tradition, le classique, Mahler y découvre toute une vie ignorée; il les rejette violemment sur la brèche, à leur place de précurseur et s'en fait à lui-même des compagnons d'avant-garde (Ritter 1906 : 247).

La musique de Mahler se reconnaît à sa densité; en témoignent le gigantesque effectif de l'orchestre mahlérien, la durée anormalement longue de certains mouvements ou œuvres, la profusion des matériaux ou ses fulgurances rythmiques, thématiques et harmoniques. Il y a quelque chose d'excessif dans cette musique aux couleurs de crépuscule et Mahler se heurte à des critiques parfois acerbes, auxquelles il semble rétorquer avec ces mots devenus célèbres : « Meine Zeit wird kommen $»^{10}$ (« Mon temps viendra »). Ritter, comme s'il avait pressenti le tournant décisif qui s'amorçait, entreprend de défendre et de promouvoir ce langage neuf qui ne pouvait que le séduire :

Oh ! comme elle est peu faite pour les académies, les conservatoires, les salons, le goût français, les manières du grand monde, les cours. Elle s'adresse à l'humanité directement, en dehors des institutions, des classes et des modes. Elle ne japonisait pas, elle ne goncourdisait pas, elle ne scolisait ${ }^{\text {II }}$ pas, elle n'archaïsait pas. Elle vivait. Et surtout elle pensait à la mort sans effarement (RpI5 I9II, cité par Meylan 2000 : 272).

\section{La critique musicale: entre témoignage, musicographie et musicologie}

La critique musicale de Ritter est fortement subjective et appartient davantage à la critique musicographique (courante à cette époque) que musicologique. Cela dit, Claude Meylan souligne « la sagacité presque prophétique de ces jugements » (Meylan 2000: $4^{\text {e }}$ de couverture), ce dont l'intéressé se flattait déjà : " [Mahler] se sait compris et s'étonne même plus d'une fois de certaines perspicacités auxquelles il ne s'attendait pas de la part d'un Welsche $^{12}$ » (Rp 31, I945 cité par Meylan 2000 :36).

Ritter se concentre principalement sur les symphonies (qui représentent certes une grande partie de l'œuvre dont il est question, mais non sa totalitér's), et chacune des dix symphonies de Mahler fait l'objet d'au moins une publication de sa part. Dans le fonds Ritter déposé aux Archives littéraires suisses à Berne se trouve une analyse détaillée de la symphonie $\mathrm{n}^{\circ} 7$ intitu-

Io Propos rapporté dans son journal par Natalie Bauer-Lechner, amie proche de Mahler.

II Allusion à la Schola Cantorum de Vincent d'Indy, alors très influente.

I2 Personne originaire de Suisse romande, pour les germanophones.

I3 L'œuvre de Mahler se compose également de musique de chambre mais surtout de Lieder dont les importants cycles Lieder eines fahrenden Gesellen, Des Knaben Wunderhorn, RückertLieder ou encore les Kindertotenlieder. 


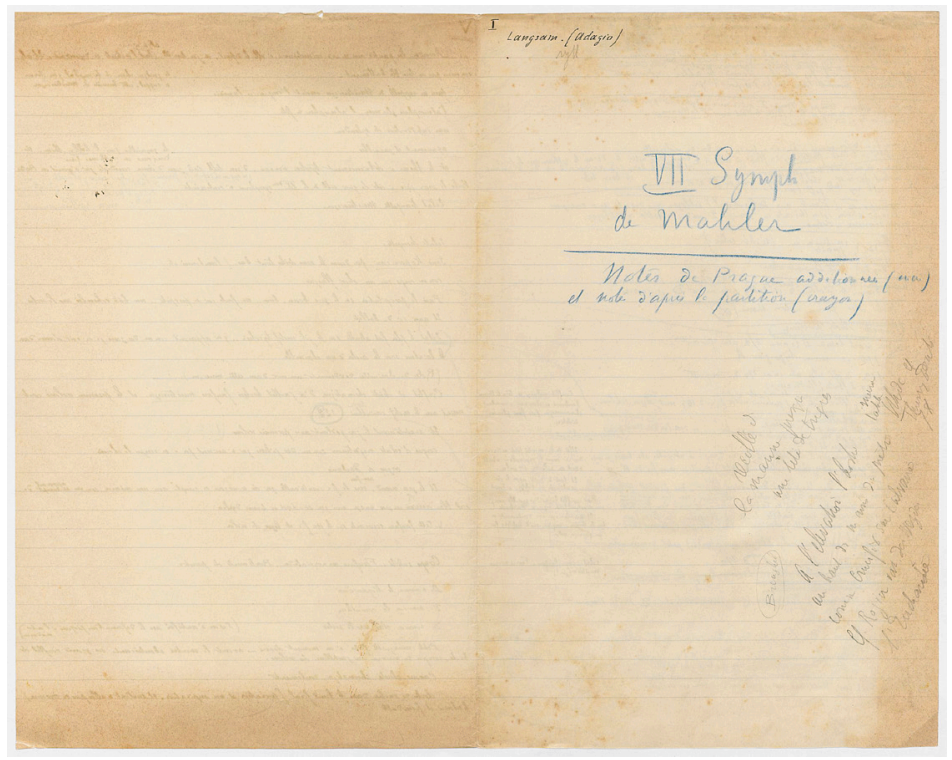

Fig. I : VII Symph de Mahler. Note de Prague additionnées (encre) et notes d'après la partition (crayon)

lée ${ }^{14}:$ «VII Symph de Mahler. Note de Prague additionnées (encre) et notes d'après la partition (crayon) » (fig. I). Ce document de dix pages grand format ressemble fort à une méthode systématique d'analyse. Ritter a-t-il procédé de la même manière pour toutes les symphonies? Qu'en est-il de son rapport aux autres compositeurs actifs à cette époque ? On sait que Ritter rencontre Bohuslav Martinů et Béla Bartòk en 1928, Igor Stravinsky en 1930 et qu'il s'intéresse à Richard Strauss depuis les débuts de ce dernier en I9Io. En outre, les noms d'Arnold Schönberg ou d'Alban Berg apparaissent çà et là dans les chroniques de Ritter, confirmant le fait qu'il connaissait également ces compositeurs, alors avant-gardistes. Seule une exploration approfondie des archives nous permettrait de répondre à ces questions essentielles.

Revenons pour l'heure à l'analyse de la symphonie $\mathrm{n}^{\circ} 7$ de Mahler. Il s'agit d'une multitude de notes dont l'organisation démontre que le critique procède par recoupement entre les notes prises à l'écoute et l'étude de la partition, manuscrit de l'analyse de la symphonie $\mathrm{n}^{\circ} 7$ de Gustav Mahler par William Ritter (fig. 2). Les notes de l'audition sont composées en premier lieu de descriptions et d'observations (quel instrument intervient et comment) qui témoignent de l'attention et de la concentration de Ritter : « La

I4 Document provenant directement des ALS à Berne, boîte nº 388, cote A V 6-I2. Toutes les citations qui suivent sont issues de ce document, sauf indication contraire. 


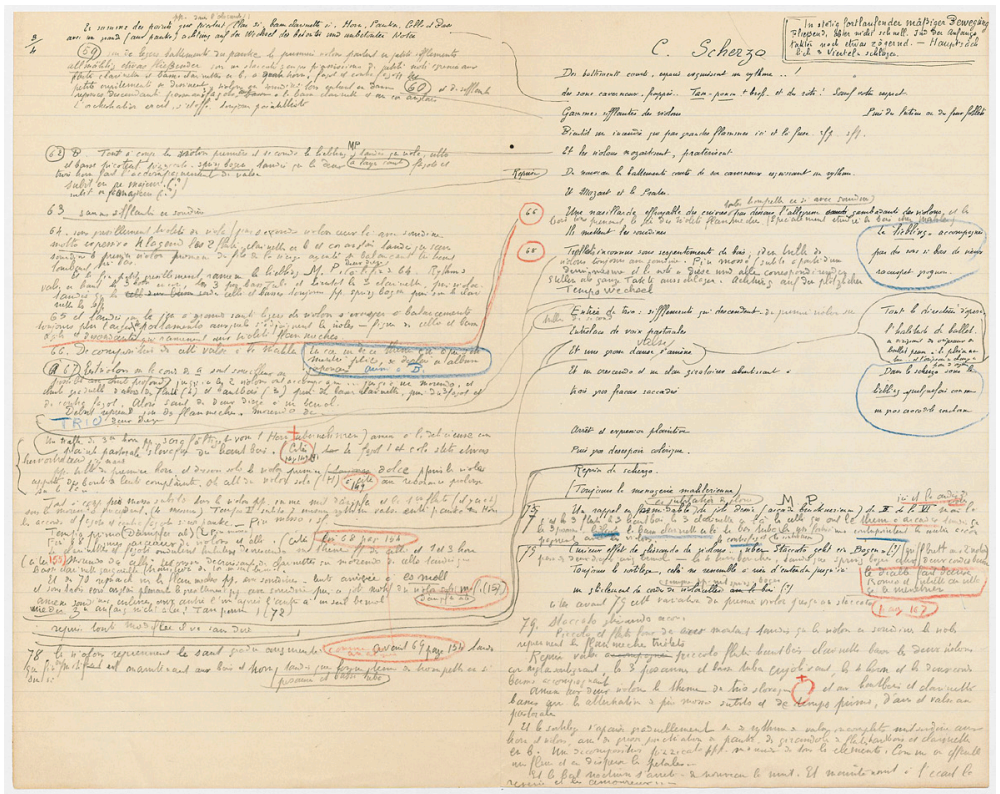

Fig. 2 : manuscrit de l'analyse de la symphonie $\mathrm{n}^{\circ} 7$ de Gustav Mahler par William Ritter

cymbale est frappée avec la baguette rembourrée d'éponge ", « la harpe est écrite dans les sons harmoniques et leur façon de résonner une octave plus haut [...] », « les violons, frappés avec le bois de l'archet », « ils contiennent la nasale sourdement, avec un mugissement du nez sur la bouche fermée ». Conclusion du critique : "Il faut que chaque instrument ait fait au moins une ou deux fois ce pourquoi il n'est pas fait » (Ritter $1906: 257$ ). D'autre part, Ritter livre ses impressions, qui relèvent dès lors de l'interprétation, reconstituant en quelque sorte le programme abandonné par Mahler ${ }^{15}$ : " belle chute sentimentale ", " carnage, bataille avec les coups de trique ", " espèce de lente oraison funèbre ", « un incendie qui par grandes flammes ici et là fusent ». Déjà il analyse et tisse des liens en puisant dans sa réserve d'images, de mots et de sons : «Brucknérien, aussitôt mahlérien », « les violons mozartisent, praterisent ${ }^{16}$ », « un plein nocturne klimto-shakespirien ».

Le monde musical mahlérien est pour Ritter un terrain sensuel de recherche, de découverte et de création. Happé par cette musique évocatrice,

I5 Un programme est un texte censé guider l'imagination des auditeurs lors de l'exécution d'une œuvre instrumentale. Le xix ${ }^{\mathrm{e}}$ siècle est l'âge d'or du genre qu'est la musique à programme et dont Berlioz et Liszt sont parmi les plus fameux représentants. Il ne s'agit toutefois pas de subordonner la musique au texte, qui reste secondaire. Mahler abandonne les programmes dès la symphonie $\mathrm{n}^{\circ} 5$.

I6 Néologisme de Ritter. Le Prater est un grand parc au cœur de Vienne en Autriche. 
le critique relève ses impressions et note ses hypothèses. Il prend même soin de rapporter quelques réactions observées dans le public, vivant le concert comme une expérience totale. Significativement, il écrit à l'encre ${ }^{17}$, comme si c'était là des faits incontestables qu'il n'y aurait nullement besoin de reprendre. Plus tard, partition à l'appui, au crayon cette fois (parfois de couleurs différentes), il revient sur ses notes, les corrige et les complète (" une grosse danse valse s'amène »), confronte l'oral à l'écrit. Flèches, ratures, mots biffés, encadrés, entourés, la pensée de Ritter est donnée à voir par le manuscrit, et elle progresse par fulgurances. Il travaille la symphonie comme pour la faire sienne : «Désormais, nous allions passer notre temps à tripoter la $4^{\mathrm{e}}$ symphonie» (Rp3I, I945 cité par Meylan 2000 : 28).

Comme l'a souligné Philippe Kaenel (I998), Ritter est imprégné par la critique baudelairienne, " partiale, passionnée et politique ", qu'il a fait sienne au fil de son contact avec les œuvres d'art. Mais s'il est d'ordinaire sûr de son jugement, il se rend très vite compte de l'incommensurabilité de l'objet d'art auquel il s'intéresse désormais en abordant Mahler :

À force d'attention et de ne rien vouloir perdre, je me trouvais comme dans ce cauchemar où une poutre, que vous êtes mis en demeure d'avaler, vous obstrue la face, et où, quand vous arrivez à vous écarter de son centre assez pour en voir le prolongement, vous la voyez se perdre à l'horizon comme un rail de voie ferrée (Ritter $1906: 262$ ).

Ritter n'est pas immédiatement convaincu et il explique:

Il me semble, par notre expérience, que, sous le coup de votre révélation, le choc en retour de la sensibilité se découvre ainsi plus sincère ! Vous semblez au premier abord contradictoire à tant d'habitudes de notre esprit, à tant d'articles de foi de notre suffisance ! Comment se rendre d'emblée à une telle révélation ? Vous êtes un monde si nouveau toujours, parfois si nouveau à force d'être ancien (RpiI, I9Io cité par Meylan 2000:232).

Ce qui nous aveuglait [...] c'était notre loyalisme envers le passé exaspéré par cette mise en capitolade de tous nos principes d'art (Ritter I906:273).

Il faut attendre la symphonie $n^{\circ} 5$ pour qu'advienne la conversion. Après celle-ci, la passion pour Mahler est sans retour, même si Ritter a besoin de temps pour progresser, symphonie après symphonie, dans l'épais et étourdissant univers mahlérien :

Nous continuerons à envisager M. Mahler comme l'un des plus dangereux adversaires de notre foi esthétique et de nos esthétiques nationales, mais nous

I7 Il nous est pour l'instant impossible de définir s'il s'agit des notes prises sur place ou d'une mise au propre de ces mêmes notes. 
dûmes bientôt reconnaître que sous les grimaces le musicien est immense et que de race le cheval sous le harnachement funambulesque (Ritter 1906:273).

Dans son compte rendu de la symphonie $n^{\circ} 4$, il confie la « lutte intérieure » qui se joue en lui, non sans que cela lui confère un certain plaisir : «Ce fut une telle lutte intérieure que - comme pour libérer mon esprit j'écrivis à Mahler » (Rp2I, I945 cité par Meylan 2000 : 28). Cette musique dérange le critique et l'oblige à élargir les contours de son entendement esthétique pour absorber ce " musicien de premier ordre », qui n'en reste pas moins à ses yeux « un des cas pathologiques les plus inattendus de l'histoire» (Ritter I906: 74) :

Quant à sa $4^{\mathrm{e}}$ symphonie, que chacun en juge comme bon lui semble ; un fait demeure avéré : qu'elle soit inoubliable. J'en appelle à tous ceux qui l'ont entendue. Bien plus, je défie le critique le plus rassis, le plus rigoureux, le plus endurci d'en parler de sang-froid; je défie enfin ceux qui le haïssent comme ceux qui l'aiment de méconnaître qu'ils y ont trouvé, bon gré, mal gré, un extrême plaisir dont au surplus il reste encore à définir la qualité (RioI, I90I cité par Meylan 2000 : 49).

En I945, à près de quatre-vingts ans et après un long silence général, Ritter revient sur ses premiers contacts avec la musique de Mahler :

Seulement nous... cette musique ne nous laissait plus dormir. Nous en restions marqués, et marqués à tout jamais. Le chemin de Damas, par foudroiement et éblouissement subits, avait été prompt évidemment. Mais ici la révélation opérait à la réflexion. Et cette réflexion qui ne nous quittait plus était simple et la plus terre à terre du monde : "Tu te révoltes contre cette musique. Tu la réprouves de toute ta sagesse, de toute ton expérience et de toutes tes convictions... Mais tu luttes contre ton plaisir... Tu essaies d'être vertueux... Au fond... tu n'aimes rien tant que cela. Tu es vaincu. Que tu le veuilles ou non, tu admires » (Rp3I, I945 cité par Meylan $2000: 28$ ).

Si Ritter utilise souvent le vocabulaire musical pour parler de la peinture, avec laquelle il s'est théoriquement et concrètement familiarisé depuis l'enfance, il appelle également le lexique pictural, littéraire et architectural à la rescousse de son propos musical, le tout dans un idéal expressif qui tend au Gesamtkunstwerk ainsi qu'à la théorie du paragone (terme utilisé dans la critique d'art dès I8I7). Rien que dans les Études d'art étranger (Ritter 1906), on repère des " sonorités à la Watteau », un " nocturne böcklinien ", une " ampleur lamartinienne », un « badinage colossal presque rabelaisien » et une « cuisine goëtique » ou encore une « cathédrale de son et d'harmonie ». La vue apparaît comme un sens inséparable de la musique de Mahler, chez qui Ritter détecte « la grande ambition fastueuse du peintre d'allégorie » 
(Ritter 1906 : 270). Elle est un « kaléidoscope sonore » (Ritter I906 : 269) à la puissance évocatrice sans égal qui, en s'adressant directement à l'imagination, épouse parfaitement le symbolisme par lequel Ritter est attiré : « [Mahler] se la crée à lui-même cette nature [...]. Et l'on sent que si l'on avait à la traduire en art ce serait par les moyens picturaux extravagants dont font preuve certains tableaux sécessionnistes » (Ritter I906 : 269).

Philippe Kaenel a mis en lumière le contraste entre les positions tantôt conservatrices, tantôt avant-gardistes de Ritter, et il relève la mise en garde de ce dernier à propos de Böcklin et Segantini : "Vous verrez si d'ici à cinquante ans ces œuvres [...] n'auront pas aux yeux de nos arrière-neveux l'attrait et le charme des Greuze » (Kaenel I998 : 93). Or, Ritter fait le même genre de prophétie quant à la musique de Mahler :

Or, il faut se convaincre que viendra naturellement le jour où toutes ces "extravagances" paraîtront naturelles et n'étonneront pas plus nos après-venants de l'an 2000 que ne nous étonnent les romantismes de Berlioz, les débordements de Beethoven et de Wagner [...]. La première locomotive, la première montgolfière et le premier parapluie excitèrent les mêmes étonnements que les petites trompettes sures et le poulailler instrumental de certains moments des symphonies moderne-style en question (Ritter $1906: 258$ ).

Le critique regrette de " ne pas avoir pu être un des fanatiques de ce Richard Wagner aujourd'hui pollué comme Raphaël par l'admiration des multitudes inférieures » (Kaenel I998 : 93). Ce qu'il n'a pas pu faire avec Wagner, il l'accomplit avec Mahler. Entre le musicien autrichien et le critique suisse, la compréhension est naturelle et instantanée : «Je crois que dès la première minute, nous nous sommes compris » (Rp3I, I945 cité par Meylan 2000 : 3I). Ritter est celui qui a saisi l'intention de Mahler avant tous les autres, instaurant de ce fait une sorte d'intimité avec le compositeur qui voit en lui quelqu'un qui peut le comprendre : «Ma $6^{\mathrm{e}}$ symphonie pose d'obscures énigmes à l'auditeur, et [...] j’aimerais me savoir compris précisément de vous deux ${ }^{18}$ » (MRo4, I5.IO.I906 cité par Meylan 2000 :356). Dans le commentaire sur l'art, Ritter dont « les valeurs de critique reposent sur la notion de rupture », se sent « investi d'une mission » (Kaenel I998 : 93).

De tels artistes, véritablement hors-la-loi, en rupture avec leur temps et avec l'ordre conventionnel de l'art se situent par conséquent en dehors de la nature humaine [...]. Ils sont autres et en avance sur leur temps. Le critique d'avantgarde, semblable à un oracle, prétend les comprendre naturellement [...]. Comprendre ces artistes d'exception (« qu'à force d'aimer et d'admirer j'ai le droit d'appeler miens »), les étudier, c'est un peu les posséder en partageant une partie

I8 Ritter et son ami Montandon. 
de leur mystère. De fait, le critique est enclin à s'identifier naturellement à la lutte de l'artiste pour la reconnaissance et tend à privilégier les œuvres hermétiques (Kaenel I998: 93-94).

Mahler, ce génie juif, est donc suffisamment « hors-la-loi » pour attirer l'attention de Ritter. Il y a comme un goût d'interdit dans cette musique si neuve et mystérieuse, donc si attirante. D’autres comme lui y viendront, certes, mais il est le précurseur : «Il n'y a pas de clan mahlérien. De langue française, je suis le seul. Je crois bien haut, je mène ce grand bruit, je le voudrais bien. Nous sommes tous des convertis qui d'abord nous sommes cabrés » (Rpio, I9IO cité par Meylan 2000 : 224).

Ritter se dit « à l'affut de toutes nouveautés musicales » (Rp3I, I945 cité par Meylan 2000 : 24) et se cherche un « dieu nouveau » (Ritter $1906: 260$ ). Grâce à la musique de Mahler, le Neuchâtelois prend conscience d'être parfois en décalage avec son époque, mais montre également qu'il sait reconnaître le génie quand il l'entend :

Le résultat est atteint, c'est-à-dire que notre âme a été secouée, tortionnée et charmée comme jamais je n'aurais crû qu'il lui fut encore possible de l'être à mon âge et par des musiques postérieures à celle de mes grands enthousiasmes périmés. Qu'importe que cela vienne avec les pires rakious frelatés du fond des alambics de Galicie ou des Ghettos d'Allemagne, qu'il y ait ou non du satanisme dans le cas de cette musique sensationnelle et féérique, qu'elle soit faite de toutes les corruptions de notre temps, elle va avec le siècle; elle le représente fidèlement. Autrement que comme signe du temps, je n'approuve pas l'œuvre de M. Mahler, mais j'en jouis éperdument. Faut-il en avoir honte? (Ritter 1906 : 275).

Ritter abdique donc : « Ces pages sont l'acte par lequel moi catholique, moi traditionaliste, moi antisémite, je rends les armes devant l'œuvre de ce sorcier juif nietzschéen » (Ritter 1906, 288). Il relève néanmoins souvent la lutte qui se joue entre sa raison et ses sens, le tout sur un ton quelque peu provocateur, une douce insolence que seul peut se permettre celui qui sait qu'il a compris ce que les autres ignorent encore : « L'audition de la troisième symphonie [...] nous confirma dans nos haines et réserves et du même coup dans notre enchantement. Il n'y avait plus à se rebiffer d'avantage contre son plaisir. L'oreille se délectait, l'esprit condamnait » (RpoI, I906 cité par Meylan $2000: 75$ ). Le critique use souvent d'oxymores pour traduire l'ébranlement de l'âme qu'il subit : "impossible d'être plus varié d'une façon plus homogène », « un monde si nouveau à force d'être ancien », « les symétries asymétriques » (Ritter 1906:28I).

Finalement, et même paradoxalement, Ritter fait preuve d'un dévouement quasi apostolique vis-à-vis du compositeur, avec toute l'abnégation et 
la dimension sacrificielle que cela sous-entend. Il faut mériter Mahler, l'aimer et, pire encore, accepter que d'autres l'aiment ensuite :

Ô Maître, Maître ! Nous vous aimons d'une façon telle que notre premier mouvement c'est, égoïstes, de nous souhaiter très peu, une très petite troupe de disciples à ainsi vous aimer ! Et pourtant chacun de nous le sait, il faut que ce soit la terre entière [...] ! Qu'ils applaudissent, qu'ils crient de joie, il me semble, pardonnez-le-moi, que vous serez moins à nous! Qu'importe que nous en souffrions si vous accroissez votre gloire. Ou bien qu'ils sifflent et qu'ils hurlent, nous serrerons les rangs. Et nous aurons des cœurs de Véronique à offrir à votre ensanglantement par les épines [...]. Nous passerons silencieusement et nous marcherons, fervents, les yeux sur la grande étoile nouvelle, la comète flamboyante que vous avez allumée à notre ciel [...]. Je le dis surtout pour que vous sachiez combien nous aimerions souffrir, souffrir pour vous [...] (Rp I9IO, cité par Meylan $2000: 23 I$ ).

Cette « malédiction rittérienne », dégagée par Philippe Kaenel à l'égard de Böcklin et Segantini, reste hautement valable pour Mahler :

Comme tout écrivain d'art contemporain, Ritter vit un dilemme constant, car le privilège de cette communion artistique [...] est menacé par son rôle même de critique d'art, c'est-à-dire de divulgateur, de vulgarisateur. En effet, si son prosélytisme porte ses fruits, l'admiration populaire risque aussitôt de corrompre la jouissance élitaire de l'œuvre (Kaenel I998 : 94).

Ritter est mû par la foi du converti, une foi d'autant plus solide que la conversion a été tardive. Il compare du reste la foudroyante expérience de l'audition de la symphonie $n^{\circ} 5$ à la conversion de saint Paul, dans un acte de contrition : "Le siffleur de Munich que je fus a trouvé là son chemin de Damas » (Ritter I906:282).

\section{L'insurmontable différence : identification et mysticisme}

«Enfin ces pages vous donneront la joie triomphale d'être célébré par un parfait antisémite !! »(RM I2 (?).03.1906 cité par Meylan 2000 : 35I). Ce sont là les mots que Ritter choisit de joindre à l'exemplaire des Études d'art étranger ${ }^{19}$ offert au compositeur. Mahler est juif, et Ritter un antisémite qui adore sa musique. Il lui faut donc trouver un moyen de surmonter cet obstacle :

I9 Recueil de textes ayant pour objet divers artistes paru en 1906 aux éditions Mercure de France (cfr. bibliographie). Le texte que Ritter consacre à Mahler intitulé « Un symphoniste viennois : M. Gustave Mahler » est en fait la première étude en français sur le compositeur, comme le souligne Ritter lui-même. 
Mais évidemment aussi [Mahler] sentait derrière lui toute la poussée juive d'alors. Ce qui ne nous était évidemment pas très sympathique. Mais la question d'art allait à part. Qu'un vrai musicien se manifestât et qu'il fut ce qu'il voulait, peu importe! Nous ne lui demanderions jamais que sa musique [...] ! Je suis décidé à ne point faire un pas pour m'approcher de lui. L'homme est d'un autre clan, d'un autre parti ; l'homme ne me sera rien. En revanche, le musicien m’appartient (Rp3I, I945 cité par Meylan 2000 : 25).

En distinguant l'homme, juif, du musicien, génial, Ritter peut sereinement s'approcher au plus près du compositeur : «Car mon âme d'aujourd'hui est tellement faite de la vôtre, faite de ce que votre musique y a mis » (RpII, I9IO cité par Meylan 2000 : 232). Si Ritter possède le musicien, l'inverse est valable : "Je n'oublierai jamais le regard que [Mahler] me lança, aussi de bas en haut [...]. Ce ne fut qu'un coup d'œil décisif, qui jugeait son homme [...] et qui prenait possession »(Rp3I, I945 cité par Meylan 2000 : 3I). Le romancier incompris, exilé, s'identifie au musicien et se reconnaît en Mahler, exilé lui aussi, chef d'orchestre célèbre mais compositeur encore difficilement accepté. Ritter est dans le bon état d'esprit pour recevoir cette musique, dont la matière même semble lui correspondre : des thèmes parfois modestes, folkloriques ou populaires sont présentés avec lyrisme, extravagance, densité et puissance, mêlant grotesque et sublime, simplicité et grandeur. Donnons en exemple le troisième mouvement de la symphonie $\mathrm{n}^{\circ} \mathrm{I}$, qui commence par le début de la chanson populaire « Frère Jacques » mais présenté en mode mineur, ce qui transforme radicalement l'impression de l'auditeur et le déboussole. Le compositeur se joue-t-il de nous ? Pour Ritter, c'est le « cirque dans la cathédrale » (Ritter I906 : 273). À ce propos, citons Theodor W. Ador$\mathrm{no}^{20}$ :

[Les symphonies de Mahler] étalent sans vergogne ce que tout le monde a dans l'oreille : bribes de mélodies venues de la grande musique, chansons populaires rebattues, rengaines et refrains à la mode (1976:58).

On a toujours perçu avec une irritation particulière, comme une contradiction, ce mélange chez [Mahler] de naïveté et d'absence de naïveté : la physionomie d'une musique dans laquelle les tours populaires les plus connus se chargent de significations, alors qu'inversement les hautes prétentions de la musique symphonique ne n'y sont pas un instant remises en question [...]. La matière musicale est prosaïque, le discours sublime (Adorno 1976 : 95).

Ce contraste entre extrême simplicité du matériau et grandeur des moyens est une des principales critiques adressées alors à Mahler, mais c'est

20 Theodor W. Adorno (1903-1969), philosophe, sociologue, musicologue et compositeur allemand, élève d'Alban Berg. 
précisément ce qui plaît le plus à Ritter, parce que cela répond à son besoin d'authenticité, de ce quelque chose qui n'aurait pas encore été avili par la multitude. Le Neuchâtelois va plus loin que l'amateur éclairé qui voyage pour assister aux concerts, plus loin que le critique élogieux : il devient mystique et s'enfonce avec délectation dans une production musicale qui éveille son imagination et ses sens. Ces compositions, au potentiel symbolique immense, s'apparentent pour Ritter à un espace de création illimité ouvert devant lui. Il y a quelque chose de l'ordre du sublime romantique dans ce vertige, cette jouissive frayeur provoquée en lui par la démesure de l'œuvre ; l'homme est paralysé, sa raison est emprisonnée : «J'ai entendu cette symphonie à peu près hypnotisé sur la personne du compositeur et dans un état de stupeur presque voisin de l'abrutissement » (Ritter I906 : 262).

Peu à peu, Ritter perd tout recul critique : Mahler est selon lui au-dessus de tout et de tous, et pour toujours. Le vocabulaire se fait de plus en plus mystique. Inaccessible, cette musique flotte entre ciel et terre, paradis et enfer, dieu et diable, salut et damnation. Ainsi, dans les Études d'art étranger, Bruckner, « le grand catholique méconnu », professeur de Mahler, est le « saint qui a engendré ce démon ». Mahler est « le symphoniste qui tente Dieu », « le sorcier » qui « mériterait encore d'être brûlé vif, s'il ne se consumait pas lui-même dans le nid de flammes et de serpents ». Mais il est aussi « un astre de première grandeur » à l'origine d'une musique « messianique », «l'homme de douleur de la musique », « la plus grande illumination de notre vie », le « dieu nouveau », « le prophète sous sa seule modalité moderne possible » qui «s'étourdit d'activité pour ne pas entendre continuellement le cri de détresse de l'humanité ». Ses symphonies sont « comme des sortes de messes pontificales de notre vie intérieure ", dirigées à l'aide de " ce bâton de chef d'orchestre qui, entre ses mains, devient sceptre incantateur de grand empire mystique ». L'amour de l'apôtre Ritter culmine dans ce texte écrit en l'honneur du cinquantième anniversaire du compositeur et dont on peine à croire qu'il a été publiée ${ }^{21}$ :

Oh ! sang de toute douleur, qui est une crucifixion [...]. Je fus cet oiseau gris, ô Maître, au cœur rougi du sang de votre œuvre. L'épine de la petite rose rouge défunte, du petit rosier d'amour dont fut tressée votre première couronne d'épines, c'est d'elle que j'ai sur mon front la petite tache rouge à jamais (RpII, I9IO cité par Meylan 2000 : 235).

2I In La Voile latine, I9Io. 


\section{Les échos intimes: correspondance et journal}

Les écrits intimes de Ritter forment une masse gigantesque. On compte au total plus de cinquante mille ${ }^{22}$ lettres écrites ou reçues (Ritter gardait des copies des lettres envoyées) dont vingt-quatre adressées à Mahler, et quinze reçues de lui. Le journal que Ritter tient jusqu'au I3 mars 1955, soit deux jours avant sa mort, couvre toute sa vie, pour un total d'environ cinq cents carnets.

Dans ses lettres à Mahler, Ritter exprime son admiration pour le musicien, l'interroge quant aux œuvres à venir, demande des partitions originales afin de les étudier, fait le compte rendu de concerts où il a entendu les œuvres du Maître et le tient au courant de ce qui a été joué, par qui et de quelle manière :

Veuillez m'écrire où et quand il y aurait possibilité d'entendre votre musique, sous votre direction, avant votre départ pour l'Amérique [...]. L'autre jour, Lasalle a donné ici votre $4^{\text {e }}$. Cela n'a pas été aussi totalement mauvais que les Münchner [Neuste] Nachrichten l'ont dit. Votre bon ami le Dr Louis en a cependant profité pour déclarer, comme toujours, que c'était « votre plus mauvaise symphonie »! La dernière qu'entend ce bon Apôtre est toujours la pire !! (RM 27.08.1909 cité par Meylan 2000 : 380).

Hyperactif, Ritter rapporte, analyse, critique. Pour le compositeur, il est une précieuse source d'information quant à la réception de son œuvre, et un interlocuteur constamment disponible pour des échanges philosophiques sur la musique et l'art en général. Mahler prend toujours la peine de répondre aux lettres de Ritter, qui conserve pieusement cette correspondance. Au fil du temps, les formules d'adresse changent pour aboutir à celle conjuguant le mieux l'admiration et l'affection, celle qui exprime le respect dû au génie tout en soulignant la proximité que Ritter, l'élu, entretient avec lui : " Cher Maître et Ami ». Quand Mahler tarde à répondre, Ritter est pris d'angoisse, comme dans cette lettre datée de l'année 1907 (année noire pour Mahler, marquée par la mort de sa fille, la perte de son poste de directeur de l'opéra de Vienne et le diagnostic de son insuffisance cardiaque) :

J'espère que rien ni personne ne vous a fâché contre moi ! Vous avez dans votre voisinage M. Nebdal qui, dit-on, ne m'aime guère. Lui je comprends, mais vous? Que diable vous aura-t-on dit? J'espère bien que vous n'allez pas partir pour l'Amérique sans me dire ce qui se passe. Je serais désolé de perdre votre amitié, désolé pour vous si c'était injustement, et ce ne pourrait être qu'injustement (RMo8 20.09.1907 cité par Meylan 2000 : 362).

22 Les chiffres sont issus de l'ouvrage de Claude Meylan (2000 : 8). 
Le journal est une voie d'accès plus intime à la passion mahlérienne de Ritter. Si certains passages sont écrits à la hâte, dans un style télégraphique, d'autres sont travaillés comme des scènes (parfois repris tels quels dans des textes destinés à la publication), laissant apercevoir le romancier sous le costume du critique et de l'admirateur. Il en est ainsi dans ce récit de la première rencontre avec Mahler :

Mardi 6 novembre donc. À dix heures au Kaim-Saal [...]. Un orchestre formidable est en rumeur sur l'estrade. Le célesta intrigue avec un petit jeune homme tout recueilli qui dans les parties où il n'a rien à faire écoutera la musique avec une sorte de dévotion. Sortons pour monter à la galerie par la première porte à gauche de l'estrade qui donne sur le corridor [...] ; et de là ouvrons la porte de l'escalier, que Mahler tout petit, tout noir, tout pressé nous part dans les jambes. Le temps de dire à Marcel « c'est lui »..., Marcel de se présenter comme il a été convenu puisqu'il parle l'allemand. Il nous présentera ensuite. Mais le nom de Montandon ${ }^{23}$ ne lui dit rien. «Et moi je suis William Ritter ». Alors il lève brusquement la tête et me dévisage effrontément. C'est comme si tout à coup il avait été doué d'intelligence et que ses lunettes se fussent nettoyées... Jamais je n'ai été regardé décisivement, une fois pour toutes, avec cette âpre clairvoyance qui pèse et juge... Regard juif, mais aussitôt regard plein de bonté. Je crois que dès cette minute nous nous sommes compris (Journal III, 8.II.1906 cité par Meylan 2000:399).

Soulignons le présent de narration qu'utilise Ritter pour conter l'instant précis de la rencontre «fatale et inévitable». Les phrases sont courtes, suspendues, le critique revit ce moment préparé, redouté, rêvé, qui marque le début d'une relation fondée sur la compréhension mutuelle. Les mots sont superflus, la musique seule suffit.

La mort du Maître marque évidemment Ritter, qui l'avait déjà pressentie, trois mois avant la date effective : «J'ai comme la certitude que Mahler est mort [...]. S'il devait s'en relever les facultés affaiblies, certes mieux vaut la catastrophe» (Journal VIII 26.3.I9II cité par Meylan 2000 : 416). Puis arrive le jour fatidique, le I8 mai I9II : «Voici dix ans que je vis à cette "petite lumière"... Et maintenant ce sera la nuit »(Journal VIII I3.5.I9II cité par Meylan $2000: 417$ ). Ritter perd un repère, un père, son dieu, un guide, un esprit dans lequel il se retrouvait et duquel il pouvait se nourrir. Cette dispari-

23 Ritter rencontre Marcel Montandon en Roumanie, lors d'un des nombreux séjours qu'il effectue à Bucarest entre I890 et I89I. Avec l'accord des parents du jeune homme, Ritter l'emmène avec lui en France (Paris) dans le but de veiller à son éducation. Entretenant une liaison amoureuse, ils effectuent de nombreux voyages ensemble avant de s'installer en Autriche, proche de Vienne, où ils vivent des critiques qu'ils écrivent pour divers journaux et revues. Leur relation se transforme en amitié lorsque Marcel épouse la fille du peintre Émile Isenbart, en I90o. 
tion semble accélérer son vieillissement : « Encore un des chapitres les plus importants de ma vie qui touche à sa fin » (Journal VIII 27.05.I9II cité par Meylan 2000 : 4I7). La mort de Mahler agit sur Ritter comme un révélateur de son insuffisance et il revient sur ce qu'a été son existence avec tristesse et lucidité :

Angoisse, mollesse physique, sentiment d'une vie matérielle misérable... Et au fond, tout au fond ce désespoir d'avoir perdu Mahler !... [...] J'ai été sans cesse au-dessous, non seulement de mon destin et de mes ambitions, mais de mon devoir [...]. Dieu ! Avoir aimé Mahler et n'être que ma misère !... Il faut, il faut que, pour l'amour de lui encore, je m'élève à lui... (Journal VIII 27.05.I9II cité par Meylan 2000 : 4I7-4I8).

Ritter n'a que quarante-quatre ans mais, fidèle au lien quasi télépathique qui l'unissait au compositeur, il pressent son propre déclin. Ainsi, dans la biographie dictée à Josef Tcherv, il fait débuter l'ultime tranche de sa vie, taxée de «vieillesse », en I9I4, soit quarante et un ans avant son décès ! Le critique vivait à travers Mahler, pour remplir sa mission d'apôtre et d'évangéliste, criant et écrivant partout la bonne nouvelle de l'existence de ce " dieu nouveau ». Sans œuvre à entendre et à défendre, sans cause pour laquelle se battre ni dieu pour lequel souffrir, Ritter est désemparé : "Mahler disparu, toute joie et tout soleil se sont retirés de la musique pour moi » (Journal VIII I3.05.I9II cité par Meylan 2000 : 4I7). Et quelques mois plus tard, il s'avouera orphelin :

Je me sens au cœur un grand vide, un grand malaise... Jamais je n'ai mieux senti à quel point il n'est plus là, à quel point son départ fait un trou dans ma vie [...]. En même temps qu'orphelin, je suis devenu inutile (Journal IX I6.II.I9II cité par Meylan 2000 : 4I9).

Ainsi Léon Vallas, éminent critique musical, rédacteur en chef de la Revue musicale de Lyon, écrit dans son journal, en date du I9 mai I9II :

Mahler est mort en effet la semaine dernière. Voilà une mort qui a dû affecter profondément Ritter à qui j'ai cru devoir envoyer une lettre de condoléance tout comme si Mahler était un de ses parents »(Vallas : I4I).

Puis, le 26 mai de la même année :

Je reçois deux lettres intéressantes. L'une de Ritter à qui j'avais envoyé des condoléances à l'occasion de la mort de son dieu Mahler. Il ne veut pas accepter d'écrire pour la Revue un article nécrologique et m'annonce même « qu'il n'écrira plus guère de choses sur la musique désormais... qu'il est 
pour longtemps désaffectionné de la musique, pas tant que désaffecté de la critique... ». La mort de Mahler - c'était à prévoir -, lui a donné un coup terrible. Pauvre Ritter» (Vallas : I42).

\section{La fusion de la critique et du roman}

"La musique de Mahler a le tort, si c'en est un, de n'être pas isolée de l'âme de celui qui la contemple $»^{24}$ (RpI4, I9II cité par Meylan 2000 : 265). La dimension autobiographique du travail de Mahler n'a pas échappé à Ritter, et il ne fait aucun doute qu'il se reconnait aussi en cela, lui dont la vie et l'œuvre ne font qu'un, lui qui « au travers de la critique d'art expose de manière narcissique le roman de sa vie, de ses passions, de ses errances et de ses rêves " (Kaenel I998: 78). Le critique et le romancier semblent ainsi parfois se confondre. Les innombrables notes hétérogènes conservées par Ritter, méticuleuses descriptions déployées pour saisir le réel, font penser au travail préparatoire du romancier plus qu'à celui du critique musical :

Le fait est que le seul souvenir que je garde de ces 42 minutes de musique compacte est celui de ce petit nécromane en habit mal taillé, de ce petit homme noir à la lèvre mince et rasée, à la physionomie de mauvais prêtre, d'un calme fantastique de psylle devant ses cobras, une touffe de crins au sommet d'un crâne dolichocéphale, médusant un orchestre fou, blême d'attention, presque rien que de ses yeux d'encre, aigus comme des langues de vipères et tenant en respect, excitant et apaisant les dragons déchaînés du bout de la petit baguette de coudrier des conjurations infernales (Ritter $1906: 263$ ).

Ritter aurait-il pressenti la dimension romanesque de la musique mahlérienne relevée par Theodor W. Adorno ? Pour ce dernier, les dimensions hors norme des symphonies de Mahler sont l'expression de l'émancipation de la forme admise jusque-là pour ce genre :

Le sens musical n'est plus garanti par la forme symphonique - que ce soit sous la forme d'une forte unité ou d'un contenu de vérité - [...] et la forme doit chercher ce sens. Il faut aller chercher dans une sorte de pure immédiateté musicale [...] les médiations qui vont lui permettre, en la chargeant de sens, de se justifier. La forme mahlérienne rejoint en cela, du point de vue de la philosophie et de l'histoire, celle du roman (Adorno 1976 : 95).

On s'étonne également des erreurs commises par Ritter quant à la chronologie de la vie de Mahler, fait curieux si l'on considère le culte qu'il voue au compositeur. Approximations et déplacements de dates ou d'événements

24 Notons encore une fois que pour Ritter, la musique se contemple autant qu'elle s'écoute. 
importants (comme la mort de la fille de Mahler) sont peut-être chez Ritter les marques d'une volonté inconsciente de faire corréler les faits avec son ressenti, dans une poussée créatrice. Il avait prévenu : «Le musicien m'appartient ». Le critique semble ainsi s'être approprié ces vers de Mahler chantés à la fin de la symphonie $n^{\circ} 2$ :

Dein ist, was du gesehnt!

Dein, was du geliebt, was du ges-

triten!

O glaube:

Du warst nicht umsonst geboren!

Hast nicht umsonst gelebt, gelitten!

C'est à toi ce que tu as désiré !

À toi ce que tu as aimé, ce pour quoi tu t'es battu!

Crois-le :

Tu n'es pas né en vain!

Ce n'est pas en vain que tu as vécu, souffert!

S'ils ne constituent pas une référence musicologique à proprement parler, les écrits de Ritter sur Mahler n'en restent pas moins, dans toute leur diversité, un fantastique témoignage de l'avènement du compositeur, presque au jour le jour. Comme le souligne Jean Balissat ${ }^{25}$, Ritter « nous rend contemporains du grand compositeur autrichien»(Meylan 2000). Pour Kurt Blaukopf ${ }^{26}$, Ritter appartient à l'avant-garde mahlérienne et ses textes, bien qu'étant de « trop empathiques déclarations d'amour», sont incontournables :

Les épanchements de ce converti occupent une place unique dans la littérature étrangère de cette époque. William Ritter était capable de lire dans l'âme de Mahler, ce qui n'était accessible qu'aux amis les plus intimes du compositeur [...]. Son vaste horizon artistique et musical permettait à Ritter de mieux préciser le lieu historique de l'art de Mahler que ne pouvaient le faire la plupart des commentateurs allemands et autrichiens de cette époque [...]. Il avait compris que Mahler n'était pas un romantique attardé, et qu'il n'était pas non plus le traînard d'un peloton qui avait eu jadis à sa tête Berlioz, Liszt et Wagner mais qu'il était au contraire un novateur absolu qui avait entrepris de conquérir le xx ${ }^{\mathrm{e}}$ siècle (Blaukopf 1979 : 205).

25 Jean Balissat (1936-2007), compositeur et chef d'orchestre suisse.

26 Kurt Blaukopf (1914-I999), sociologue autrichien s'étant beaucoup intéressé à la sociologie de la musique. 
Malgré son manque d'objectivité, ou peut-être grâce à cela, cette critique d'un genre particulier, dont une grande partie reste encore à explorer, constitue donc, loin de l'anecdote, un apport réel tant pour les musicologues et les historiens de la musique que pour les amoureux de Mahler. Tous liront avec passion cet ultime roman rittérien, un roman empreint de mysticisme dont le dieu Mahler est le héros et dans lequel Ritter joue son propre rôle: un roman qui parle d'une musique venue changer le monde.

\section{Bibliographie}

Adorno, Theodor W., Mahler, une physionomie musicale, Paris, Les Éditions de minuit, 1960.

Beltrando-Patier, Marie-Claire (dir.), Histoire de la musique, Paris, Bordas, 2004.

Blaukopf, Kurt, Gustav Mahler, Paris, Robert Laffont, 1979.

de la Grange, Henry-Louis, Gustav Mahler, Paris, Fayard, 2007.

Kaenel, Philippe, «William Ritter : un critique cosmopolite, böcklinien et anti-hodlérien ", Revue suisse d'histoire, vol. 48, n I, I998, pp. 73-98.

Matter, Jean, Mahler le démoniaque, Lausanne, L'Âge d'homme, 1959.

Meylan, Claude, William Ritter, chevalier de Gustav Mahler. Écrits, correspondance, documents, Berne, Peter Lang, 2000.

Ritter, William, Études d'art étranger, Paris, Mercure de France, 1906.

—. «Souvenirs sur Anton Bruckner», Feuilles musicales, IO, 1956, pp. I89-19I.

Vallas, Léon, Journal d'un critique musical lyonnais (1907-1940), introduit par Philippe Roger et Jérôme Dorival, Lyon, Symétrie, 2018.

Vogel, Laura, «William Ritter et le wagnérisme: Egyptiacque, une paraphrase wagnérienne? », Versants, 66:I, 2019.

Walter, Bruno, Gustav Mahler, Paris, Le Livre de poche, 1970. 